\title{
3D visualized technique for coalbed methane integrated surface and underground drainage
}

\author{
DU Xinfeng \\ Xi'an Research Institute of China Coal Technology \& Engineering Group Corp, \\ Shaanxi Xi'an, 710054, China \\ duxinfeng@cctegxian.com
}

\begin{abstract}
According to the different features of 3D elements in the coalbed methane (CBM) surface and underground drainage system in the coal mining area, and adopting the 3DMAX static modeling of the drainage equipment and the dynamic modeling of the geological body based on the borehole data, the 3D model of the CBM drainage element in coal mining area is realized. Applying the techniques such as the original data analysis to control the accuracy of the interpolation algorithm, and the blanking and cutting of the window, the visualized functions of the $3 \mathrm{D}$ elements such as the multiple-angle observation, magnification, roaming, rotation, translation, stack, peel, transparency and texture treatment are realized.
\end{abstract}

Keywords- coalbed methane (CBM); integralized drainage; $3 D$ modelling and visualization

\section{I.INTRODUCTION}

In the aspects such as the development pattern, time and space layout and so on, the surface development and the underground drainage of CBM are both mutually independent, and interdependent and mutually affected. How to effectively coordinate the surface development and the underground drainage of CBM in coal mining area and fully develop the superiorities, e.g., the high efficiency of the CBM surface ahead drainage and the timely and conveniently underground suction of the mine gas, they are the problems needed urgently to resolve at present.

The 3D visualized management system for CBM integrated surface and underground drainage utilizes the functions of $3 \mathrm{D}$ visualized technique to display lively and imaginably the space elements in the CBM surface development project and underground mine gas suction project, such as the formation, borehole, roadway, gas suction pipe line and suction station, etc., and based on these project elements which carrying lots of the attribute data, applying the coal and CBM geology, CBM assessment and development theory, as well as the gas occurrence regularity and drainage technique, to conduct the analysis and study, and provide the support for the CBM surface and underground coordination development in the coal mining area. Through this system, it can enhance the audio-visual property and accuracy of the geological analysis to the maximum, and decrease the risk faced in the gas drainage project in coal mining area. The modelling and visualization of $3 \mathrm{D}$ elements in the coal mining area are the technical difficulties in the system development.

\section{II.SYSTEM FRAMEWORK}

In the coal mining area, 3D elements related to the CBM surface development and underground gas suction are mainly the surface buildings, boreholes, formation, underground roadway and mine gas suction system, of which, the mine gas suction system includes the underground moving suction system (pipe, water draining device, anti-air return device and water sealed explosive-proof box, etc.) and the surface permanent drainage system (drainage pipe and drainage station, etc.). The management of 3D elements adopts the data organization mode faced to the entity and topological relation, each element consists of 3D geometric property and attitude, and many other elements also can constitute a new element. The data management includes two paths, one conducts the alternation with the special data management module through ADO mechanism; another one visits the external file through $\mathrm{I} / \mathrm{O}$ mode and conducts the reading and storing. In addition, the system also includes 3D geological modelling module, expert intelligent decision module, drainage design module, analysis and prediction module and one special 3D visualized assembly, and the adopted framework is shown in Fig.1.

\section{III.3D ELEMENT MODELLING}

3D elements concerned in CBM surface and underground drainage system are mainly the surface buildings, formation, borehole, roadway, gas suction equipment, etc.; based on the modelling method, it can be divided into the static modelling and dynamic modelling, of which, the static modelling includes the surface buildings and gas suction equipment, and the dynamic modelling includes the formation, borehole and roadway, etc. 


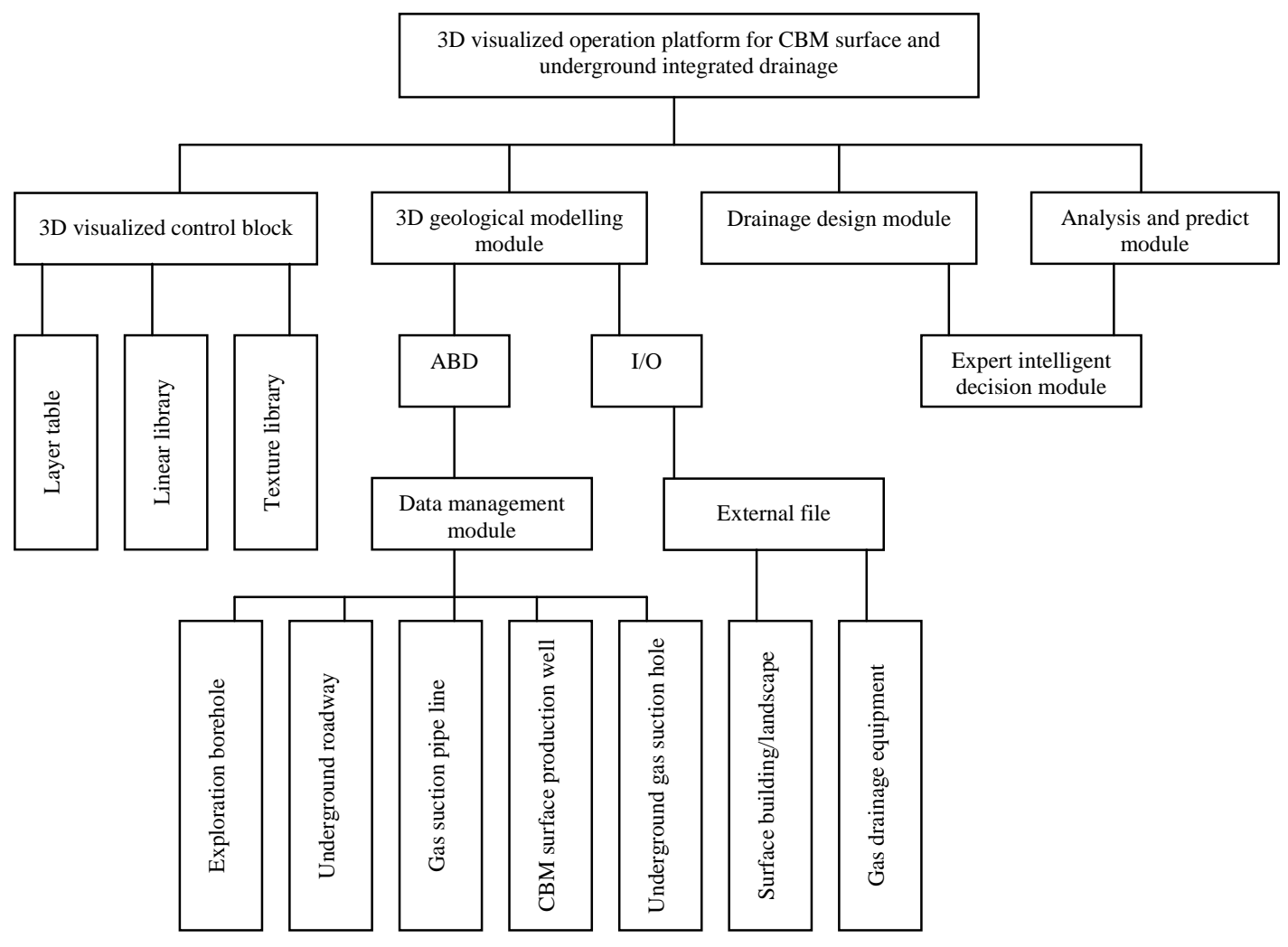

Fig.1 Design framework of 3D visualized management system for CBM integrated surface and underground drainage

The surface buildings and gas suction equipment have the features such as the meticulous and regular expression and once forming, etc., during the modelling, at first, the objects and targets are photographed, it requires the clear texture, lesser deformation, bright and saturated colour, less data quantity and decreasing shelter, if necessary, it conducts the simple image treatment. Then, in 3DMAX it creates a 3D model, again, applying 3DGSLib control block of the system and through analyzing 3D file format to read the model data, and the data are integrated. Finally, by a series of rotation, translation and reduction and magnification operations, it is set up into the system model.

Due to the complexity and variety of its 3D space, the geological body (here it refers mainly the formation and ground surface), it is difficult to design a kind of the data model or the data structure for describing the complicated 3D space phenomena. From the basic element of modelling and the modelling method, it can be seen that 3D space data model could be summarized as three categories: the Facial Model expressed by the face, the Volumetric Model expressed by the volume, and the Hybrid Model expressed by the mixture ${ }^{[1]}$. From the data description form, it also can be divided as three kinds: grid, vector and mixture of both grid and mixture. In recent years, many internal researchers provided the better method in which the limited data are applied combined with the mathematical model description to construct 3D complicated geological modelling, such as the super-volume element model method, NURBS auxiliary geological modelling technique, polyhedron expression and interactive edition method of blocky geological body, and 3D geological modelling method based on triangular prism, etc. ${ }^{2-5]}$.

In the practical application, 3D space data model should take the direction, simplicity and practicality as the prerequisite. The drilling exploration is the main mean for the geological exploration department acquiring the formation data, and it has the features that it is overall distributed in the minefield with the even interval, etc. The formation and ground surface in the 3D visualized expert system for CBM integrated surface and underground drainage apply the modelling aspect which is based on the borehole data (surface exploration borehole, CBM parameter borehole and underground mine gas suction hole). At first, this method accords the location of the borehole coordinate on 2D plane to conduct the triangulation and establish the modelling triangular grid, and then, the modelling of triangular grid is decomposed to the formation correlation and geological body simulation in both directions: parallel to the formation extension direction and perpendicular to the formation extension direction. Especially, in the display of the geological body surface, based on the different elevations, the system sets up the 
variation of colour and brightness, and then it enhances the stereoscopic effect of the visual sense.

In fact, due to the great complexity (e.g., the fold, fault and hiatus are occurred in coal seam) and the multi-resolution of the geological phenomena, it is unrealistic that it tries to adopt one modelling method to fully and automatically resolve all coal seam modelling problems; it often needs to adopt multiple treatment methods based on the requirement, including the suitable artificial interference.

\section{IV.VISUALIZATION OF 3D GEOLOGICAL BODY}

In the $3 \mathrm{D}$ visualization process of the geological body, the most important work is to acquire the 3D coordinate information data of the geological body, it is transformed to the screen 2D coordinate, and further it is displayed on the screen. During acquiring the data, at first, to conduct the analysis on the GRID DEM data formed by the interpolation, if the accuracy is too low, the original data are refining by different interpolation algorithms; if the accuracy is too high, considering reducing the procedure running load, the data are sparse-extracting. During the display, the data are conducted the projection transformation to calculate the screen coordinate it displayed on the screen. In order to reflect distinctly the transformation of the brightness and colour of the formation surface, combining with the setup of the light source location and illumination direction of the scene, the system calculates the normal component of the nodal point and surface, and on this base it obtains the colour and grey scale of each image element on screen. In addition, the scene also is conducted the blanking and cutting treatment, the blanking is conducted for really showing the depth relation of the object, the cutting is conducted for not displaying the object out of the current window, and raising the procedure running efficiency.

The visualization of $3 \mathrm{D}$ visualized expert system for CBM integrated surface and underground drainage is completed mainly by the layer table, model table, texture table, symbol library, linear library and a special display operation platform, and the visualized functions such as the multiple-angle observation, magnification, roaming, rotation, translation, stack, peel, transparency and texture treatment of the surface buildings, formation (including coal seam), underground roadway and mine gas drainage system in the coal mining area are realized.

\section{V.CONCLUSION}

According to the different characteristics of the gas drainage element in coal mining area, and applying the geological body dynamic modelling and the 3D MAX static modelling based on the borehole data, it both enhances the $3 \mathrm{D}$ effect of the model and raises the modelling efficiency. Through the techniques such as the original data analysis to control the accuracy of the interpolation algorithm, and the blanking and cutting of the window, the visualized functions of the gas drainage system in coal mining area are realized.

\section{REFERENCES}

[1] Chen Jian. Modelling and analysis study on 3D formation information system. [Ph.D. Dissertation]. Wuhan Rock and Earth Mechanical Institute of the Chinese Academy of Sciences, 2001.2. (in Chinese).

[2] Wu Qiang, Xu Hua. Study on 3D geological modelling and visualization method [J]. China Science (D compile), 2004, 34 (1): 54-60 (in Chinese).

[3] Chen Shaoqiang, Li Qi, Miao Qianjun et al. Edition method of 3D geological model combined with victor and grid [J]. Journal of Computer Graphic Auxiliary Design and Graphics, 2005, 17 (7): 1544-1548 (in Chinese).

[4] Zhong Denghua, Li Mingchao, Wang Gang et al. NURBS auxiliary modelling of complicated geological body and visualization analysis [J]. Journal of Computer Graphic Auxiliary Design and Graphics, 2005, 17 (2): 284-290 (in Chinese)

[5] Zhang Yu, Bai Shihua. 3D geological modelling method based on triangular prism element and its application [J]. Journal of China Image and Graphics, 2001, 6 (3): 285-290 (in Chinese). 